\title{
TRANSITIVITY OF FAMILIES OF INVARIANT VECTOR FIELDS ON THE SEMIDIRECT PRODUCTS OF LIE GROUPS
}

BY

\author{
B. BONNARD, V. JURDJEVIC, I. KUPKA AND G. SALLET
}

\begin{abstract}
In this paper we give necessary and sufficient conditions for a family of right (or left) invariant vector fields on a Lie group $G$ to be transitive. The concept of transitivity is essentially that of controllability in the literature on control systems. We consider families of right (resp. left) invariant vector fields on a Lie group $G$ which is a semidirect product of a compact group $K$ and a vector space $V$ on which $K$ acts linearly. If $\mathscr{F}$ is a family of right-invariant vector fields, then the values of the elements of $\mathscr{F}$ at the identity define a subset $\Gamma$ of $L(G)$ the Lie algebra of $G$. We say that $\mathscr{F}$ is transitive on $G$ if the semigroup generated by $\bigcup_{X \in \Gamma}\{\exp (t X): t \geqslant 0\}$ is equal to $G$. Our main result is that $\mathscr{F}$ is transitive if and only if $\operatorname{Lie}(\Gamma)$, the $\operatorname{Lie}$ algebra generated by $\Gamma$, is equal to $L(G)$.
\end{abstract}

0. Introduction. In this paper we give necessary and sufficient conditions for a family of right-invariant vector fields on a Lie group to be transitive. This concept of transitivity is essentially that of controllability in the literature on control systems. In our context it can be explained as follows. Let $G$ be a Lie group with its Lie algebra $L(G)$. There is an obvious correspondence between subsets of $L(G)$ and right (respectively, left) invariant families of vector fields on $G$. If $\Gamma \subset L(G)$ we denote by $\tilde{\Gamma}_{r}$ the corresponding right-invariant family of vector fields. $X \in \tilde{\Gamma}_{r}$ if and only if $\tilde{X}_{e} \in \Gamma$ where $\tilde{X}_{e}$ is the value of $X$ at the identity $e$ of $G$. If $\tilde{X}$ is a right-invariant vector field then the one-parameter group of diffeomorphisms of $\tilde{X}$ is given by $\{\exp t \tilde{X}: t \in \mathbf{R}\}$.

Corresponding to a subset $\Gamma$ of $L(G)$ we let $S(\Gamma)$ be the semigroup generated by $\cup_{X \in \Gamma}\{\exp t X: t \geqslant 0\}$. We say that $\tilde{\Gamma}_{r}$ is transitive on $G$ if $S(\Gamma)=G$. If $\tilde{\Gamma}_{r}$ is a given subset of right-invariant vector fields, then there is an obvious corresponding subset $\tilde{\Gamma}_{l}$ of left-invariant vector fields, and $\tilde{\Gamma}_{l}$ is transitive whenever $\tilde{\Gamma}_{r}$ is. For that reason we will only consider the right-invariant families on $G$.

It is well known that a necessary and sufficient condition for $S(\Gamma)$ to have a nonempty interior in $G$ is that $\operatorname{Lie}(\Gamma)$, the Lie algebra generated by $\Gamma$, be equal to the Lie algebra of $G$ (for instance, $[\mathbf{S J}]$, $[\mathbf{K}]$ and $[\mathbf{L}]$ ). It is also well known that when $G$ is compact and connected this condition is equivalent to transitivity of $\tilde{\Gamma}[\mathbf{J S}]$.

In this paper we show that $\operatorname{Lie}(\Gamma)=L(G)$ is essentially equivalent to transitivity of $\tilde{\Gamma}$ on $G$, which is a semidirect product of a vector space $V$ and a compact group $K$

Received by the editors February 2, 1981 and, in revised form, March 24, 1981.

1980 Mathematics Subject Classification. Primary 49E15; Secondary 53A04.

(C) 1982 American Mathematical Society 0002-9947/82/0000-1058/\$04.00 
(such $G$ is always noncompact). More specifically, we let $K$ be a compact connected Lie group which acts linearly on a vector space $V$, and we let $G$ be the semidirect product of $V$ with $K$. We denote this by $G=V \times{ }_{s} K$. Recall that this means that the elements of $G$ are the elements of $V \times K$ with the group operation defined by $(v, g) \cdot(w, h)=(v+g w, g h)$ for all $(v, g)$ and $(w, h)$ in $G . L(G)$, the Lie algebra of $G$, consists of elements of $V \times L(K)$ where the Lie bracket is defined as $[(a, A),(b, B)]=(A b-B a,[A, B])$ for all $(a, A)$ and $(b, B)$ in $L(G)$ and where $[A, B]$ is the Lie bracket in $L(K)$.

This paper is divided into four sections. The first section deals with the basic definitions and elementary facts concerning Lie groups and semidirect products. When $K$ is such that $K v=\{k v: k \in K\}=\{v\}$ only for $v=0$, i.e., when $V$ admits no fixed points of $V$ except the origin then $\operatorname{Lie}(\Gamma)=L(G)$ is a necessary and sufficient condition for transitivity of $\tilde{\Gamma}$. §II explains this result. In addition it contains a criterion for $\Gamma$ which ensures that $\operatorname{Lie}(\Gamma)=L(G)$ when $K$ is semisimple, and acts irreducibly on $V$.

$\S$ III deals with the case where $V$ has fixed points under the action of $K$. If $V_{1}=\{v: K v=v\}$, then in addition to $\operatorname{Lie}(\Gamma)=L(G)$ we must require that the orthogonai projection of $C_{0}(\Gamma)$, the positive convex cone spanned by $\Gamma$, on $V_{1}$ be equal to $V_{1}$. In such a case, these two conditions are both necessary and sufficient for transitivity of $\tilde{\Gamma}$.

Finally, §IV deals with the related results and applications. This section contains a transitivity result on a semidirect product of $V$ with a Lie group $H$ which is not necessarily compact but which has a maximal compact subgroup $K$ admitting no nonzero fixed points in $V$. In such a case a necessary and sufficient condition for transitivity is that $\operatorname{Lie}(\Gamma)=L(G)$ and that the projection $\tilde{\Gamma}_{H}$ of $\tilde{\Gamma}$ be transitive on $H$.

As a way of applications we treat two somewhat distinct situations. The first deals with the regular curves in $\mathbf{R}^{n}$ and their moving frames as described by the SerretFrenet formulas. The complete differential system for a given regular curve, that is, the curve along its orthonormal moving frame, is most conveniently described on $G$, the semidirect product of $\mathbf{R}^{n}$ with $O_{n}(\mathbf{R})$. In particular, we show that the present techniques generalize the results contained in $[\mathbf{J}]$.

The second application of our results is directed to what we call affine control systems on $\mathbf{R}^{n}$ of the form:

$$
\frac{d x}{d t}=A x+a+\sum_{i=1}^{m} u_{i}(t)\left(B_{i} x+b_{i}\right)
$$

where $A, B_{1}, \ldots, B_{m}$ are $n \times n$ matrices, $a, b_{1}, \ldots, b_{m}$ are vectors in $\mathbf{R}^{n}$, and where $u(t)=\left(u_{1}(t), \ldots, u_{m}(t)\right)$ is a control function taking values in some control constraint set $\Omega$ in $\mathbf{R}^{m}$. Such systems can be regarded as projections of control systems of a semidirect product of $\mathbf{R}^{n}$ with a Lie subgroup $H$ (determined by the matrices $\left.A, B_{1}, \ldots, B_{m}\right)$ of $G L_{n}(\mathbf{R})$. More specifically, we regard $X=(a, A)$ and $Y_{i}=\left(b_{i}, B_{i}\right)$, $i=1,2, \ldots, m$, as elements of $L(G)$ and we consider control systems on the form:

$$
\frac{d g}{d t}=\left(X+\sum_{i=1}^{m} u_{i}(t) Y_{i}\right) g \text {. }
$$


If $g(t)=(x(t), h(t))$ then

$$
\frac{d h}{d t}=\left(A+\sum_{i=1}^{m} u_{i}(t) B_{i}\right) h(t)
$$

and $x$ satisfies equation (1). Our transitivity results apply to controllability of equation (2) and hence to controllability of affine systems as well.

I. The basic definitions and elementary facts. As already stated in the introduction, in this paper we will study invariant differential systems on a Lie group $G$ which is the semidirect product of a finite dimensional vector space $V$ and a compact connected Lie group $K$ which acts linearly on $V$. We shall write $G=V \times{ }_{s} K$. The Lie algebra of $G, L(G)$ consists of elements of $V \oplus L(K)$ with the Lie bracket defined by $[(x, A),(y, B)]=(B x-A y,[A, B])$ where $L(K)$ is the Lie algebra of $K, x$ and $y$ are in $V, A$ and $B$ are elements of $L(K)$ and where $[A, B]$ is the Lie bracket of $A$ and $B$ in $L(K)$.

We shall write $V \oplus_{s} L(K)$ for $L(G)$ the Lie algebra of $G$. Throughout this paper $\pi$ and $\tau$ will be the canonical projections of $G$ onto $K$ and $V$ respectively. We shall write $d \pi$ and $d \tau$ for their respective differentials: $d \pi: L(G) \rightarrow L(K)$ and $d \tau$ : $L(G) \rightarrow V$, and both maps are linear.

With each subset $\Gamma$ of $L(G)$ we will associate the family $\tilde{\Gamma}_{r}$, of right-invariant vector fields on $G: X \in \tilde{\Gamma}_{r}$ if and only if $X_{e} \in \Gamma$ where $X_{e}$ is the value of $X$ at the identity $e$ of $G$. By $S(\Gamma)$ we shall denote the semigroup generated by

$$
\bigcup_{A \in \Gamma}\{\exp A t: t \geqslant 0\} \text {. }
$$

As is well known, $S(\Gamma)$ is the positive semiorbit of $\tilde{\Gamma}_{r}$ through the identity $e$. For convenience to the reader, recall that if $\mathscr{F}$ is a family of vector fields on a manifold $M$, (complete, as is the case here), then the positive semiorbit of $\mathscr{F}$ through a point $q \in M$ is the action of the semigroup generated by $\cup_{X \in \mathscr{F}}\{\exp t X: t \geqslant 0\}$ at $q$. Here, $\{\exp t X: t \in \mathbf{R}\}$ is the 1-parameter group generated by the vector field $X$. (For further details, see [JK].)

Definition 1. $\tilde{\Gamma}_{r}$ is transitive on $G$ if $S(\Gamma)=G$.

In the sequel we assume that $\Gamma$ is a fixed subset of $L(G)$ and we seek conditions on $\Gamma$ which characterize the transitivity of $\tilde{\Gamma}_{r}$.

Definition 2. We shall say that $v \in V$ is a fixed point under $K$ if $K v=\{g v$ : $g \in K\}=\{v\}$. We shall write this $K v=v$.

\section{Transitivity for the cases where $K$ admits no nonzero fixed points of $V$.}

THEOREM 1. Suppose that $V$ and $K$ are such that $V$ admits no fixed nonzero points with respect to $K$. Then, a necessary and sufficient condition for the transitivity of $\tilde{\Gamma}_{r}$ is that $\operatorname{Lie}(\Gamma)$, the Lie algebra generated by $\Gamma$, be equal to $L(G)$.

In the proof of this theorem we will be using the following four lemmas which we state and prove first.

We first make the following important remark which will be used several times in the sequel. 
REMARK 1. $\operatorname{Lie}(\Gamma)=L(G)$ is a necessary and sufficient condition that the interior of $S(\Gamma)$ be nonempty in $G$. (For proof, see $[\mathbf{K}]$, or $[\mathbf{S J}]$.)

Lemma 1. Assume that $\operatorname{Lie}(\Gamma)=L(G)$. Then, $\pi(S(\Gamma))=K$.

Proof. Let $\Gamma_{K}$ be the projection of $\Gamma$ into $L(K)$. Since $\operatorname{Lie}(\Gamma)=L(G)$ it follows that $\operatorname{Lie}\left(\Gamma_{K}\right)=L(K), \pi(S(\Gamma))=S\left(\Gamma_{K}\right)$ where $S\left(\Gamma_{K}\right)$ is the semigroup generated by $\cup_{A \in \Gamma_{K}}\left\{e^{A t}: t \geqslant 0\right\}$. That is, $S\left(\Gamma_{K}\right)$ is the positive semiorbit of $\left(\tilde{\Gamma}_{K}\right)_{r}$ through the identity $I$ of $K$.

Now, it is well known that $\operatorname{Lie}\left(\Gamma_{K}\right)=L(K)$ is a necessary and sufficient condition such that $S\left(\Gamma_{K}\right)=K$ because $K$ is compact (for instance, [JK] or [JS]). Thus, the statement of the lemma is true.

The next three lemmas deal with the following set:

$$
T=\{(v, I):(v, I) \in \operatorname{int} S(\Gamma)\},
$$

under the condition that $\operatorname{Lie}(\Gamma)=L(G)$.

LEMMA 2. $T$ is nonempty.

Proof. Lie $(\Gamma)=L(G)$ implies by Remark 1 that int $S(\Gamma)$, the interior of $S(\Gamma)$, is nonempty. Let $(v, g) \in$ int $S(\Gamma)$. By Lemma 1 , there exists $w \in V$ such that $\left(w, g^{-1}\right) \in S(\Gamma)$. But then, since $S(\Gamma)$ is a semigroup, $(v, g) \cdot\left(w, g^{-1}\right)=(v+g w, I)$ belongs to int $S(\Gamma)$. Hence, $T$ is nonempty.

Lemma 3. For each $v \in T$ there exists an integer $N>0$ such that $(\lambda v, I) \in T$ for all $\lambda$ with $\lambda>N$.

Proof. If $(v, I) \in$ int $S(\Gamma)$ then there exists $\varepsilon>0$ such that $((1+\lambda) v, I) \in$ int $S(\Gamma)$ for all $\lambda$ with $|\lambda|<\varepsilon$. Hence $((1+\lambda) v, I)^{n}=(n(1+\lambda) v, I) \in$ int $S(\Gamma)$ for each integer $n>0$. Let $N$ be any integer with $N(1+\varepsilon)>1$. Then, the closed interval $[N, N+1]$ has the property that $(\lambda v, I) \in \operatorname{int} S(\Gamma)$ for all $\lambda \in[N, N+1]$. But by the semiproperty of $S(\Gamma)$, then the whole ray $\{\lambda: \lambda>N\}$ has such property, and hence we have the statement of the lemma.

Lemma 4. For each $(v, I) \in T$ and each $g \in K$ there exists an integer $M>0$ such that $(M g v, I) \in T$.

Proof. For each $g \in K$, by Lemma 1 there exist vectors $v_{g}$ and $v_{g^{-1}}$ in $V$ such that $\left(v_{g}, g\right)$ and $\left(v_{g^{-1}}, g^{-1}\right)$ belong to $S(\Gamma)$. Hence, $\left(v_{g^{-1}}, g^{-1}\right)\left(v_{g}, g\right)=\left(g^{-1} v_{g}+v_{g^{-1}}, I\right)$ belongs to $S(\Gamma)$.

If $(v, I) \in$ int $S(\Gamma)$ then let $M>0$ be any integer such that

$$
\left(v-M^{-1}\left(v_{g^{-1}}+g^{-1} \cdot v_{g}\right), I\right)
$$

belong to int $S(\Gamma)$. Therefore,

$$
\left(v-\frac{1}{M}\left(v_{g^{-1}}+g^{-1} \cdot v_{g}\right), I\right)^{M}=\left(M v-\left(v_{g^{-1}}+g^{-1} v_{g}\right), I\right) \in \operatorname{int} S(\Gamma)
$$


But then,

$$
\begin{aligned}
\left(v_{g}, g\right)\left(M v-\left(v_{g^{-1}}+g^{-1} v_{g}\right), I\right)\left(v_{g^{-1}}, g^{-1}\right) & \\
= & \left(v_{g}, g\right)\left(M v-g^{-1} v_{g}, g^{-1}\right)=(M g v, I)
\end{aligned}
$$

belongs to int $S(\Gamma)$. Therefore, the statement of the lemma is true.

We now turn to the proof of the theorem.

Proof. That $\operatorname{Lie}(\Gamma)=L(G)$ is a necessary condition for the transitivity follows from Remark 1. So assume that $\operatorname{Lie}(\Gamma)=L(G)$. We want to prove that $S(\Gamma)=G$. Since $G$ is connected it is sufficient to show that $S(\Gamma)$ contains a neighborhood $U$ of the identity $e$ in $G$. For then $G=\cup_{n \geqslant 1} U^{n}$, and for each $n>0 U^{n} \subset S(\Gamma)$ since the latter is a semigroup.

Let $(v, I) \in$ int $S(\Gamma)$. By Lemma 2 such a vector $v$ exists. Let $\bar{v}=\int_{K} K \cdot v d \mu$ where $\mu$ is the Haar measure on $K$ such that $\mu(K)=1$. Since $\mu$ is invariant under the translations by the elements of $R$ and since $K$ acts linearly on $V$ it follows that $\bar{v}$ is a fixed point of $K$, i.e., $K \bar{v}=\bar{v}$. By our assumption it then follows that $\bar{v}=0$.

Let $C(v)$ be the positive closed convex envelope generated by the set $\{g \cdot v$ : $g \in K\}$. Let $g_{1}, \ldots, g_{p}$ be any elements of $K$ such that any element $w \in \mathcal{C}(v)$ can be written as

$$
w=\sum_{j=1}^{p} \lambda_{j} g_{j} v \text { for some } \lambda_{1} \geqslant 0, \lambda_{2} \geqslant 0, \ldots, \lambda_{p} \geqslant 0 .
$$

In particular, since $\int_{K} K \cdot v d \mu \in \mathcal{C}(v)$ we have that

$$
0=\bar{v}=\sum_{j=1}^{p} \lambda_{j} g_{j} v \text { for some } \lambda_{1}>0, \ldots, \lambda_{p}>0 .
$$

By Lemma 4 there exist integers $M_{1}>0, \ldots, M_{p}>0$ such that $\left(M_{j} \lambda_{j} g_{j} v, I\right) \in$ int $S(\Gamma)$ for each $j=1,2, \ldots, p$. Let $M=\prod_{j=1}^{p} M_{j}$. Then, $\left(M \lambda_{j} g_{j} v, I\right) \in \operatorname{int} S(\Gamma)$ for $j=1,2, \ldots, p$. Thus

$$
(0, I)=(M \bar{v}, I)=\left(\sum_{j=1}^{p} M \lambda_{j} g_{j} v, I\right)
$$

belongs to int $S(\Gamma)$. Hence, $S(\Gamma)$ contains a neighborhood of the identity in $G$, and therefore our proof is now finished.

A particular case covered by Theorem 1 is the case where $K$ acts irreducibly on $V$. The following theorem deals with that case, and it gives a criterion which ensures that $\operatorname{Lie}(\Gamma)=L(G)$ for a given subset $\Gamma$ of $L(G)$. For that purpose we introduce the following considerations.

The group $G=V \times{ }_{s} K$ acts on $V$ in the obvious way:

$$
(v, g) x=g \cdot x+v \text { for all }(v, g) \in G \text { and all } x \in V .
$$

Since $K$ acts linearly on $V$, the preceding action is affine. For each $M=(v, A) \in$ $L(G)$ and for each $x \in V,\{(\exp t M) x: t \in \mathbf{R}\}$ is a one parameter group on $V$ whose infinitesimal generator is the affine vector field $x \rightarrow A x+v$. 
DEFINITION 3. If $\Gamma \subset L(G)$, then $\widetilde{\mathcal{Y}}(\Gamma)$ will be the set of affine vector fields on $V$ generated by $\Gamma$, i.e., $X \in \mathcal{F}(\Gamma)$ if and only if $X(x)=A x+v$ for some $(v, A) \in \Gamma$.

In the language of $[\mathbf{J K}], \mathscr{F}(\Gamma)$ is subordinated to the preceding group action and generated by $\Gamma$. If $x \in V$ we shall write $\widetilde{F}_{x}(\Gamma)$ for the set $\{X(x): X \in \mathscr{F}(\Gamma)\}$. Using the notations we then have the following

THEOREM 2. Suppose that $K$ is a connected, compact, semisimple real Lie group which acts irreducibly on a vector space $V$. Let $G=V \times{ }_{s} K$ and let $\Gamma \subset L(G)$. Then a necessary and sufficient condition for $\operatorname{Lie}(\Gamma)=L(G)$ is that

(i) $\operatorname{Lie}\left(\Gamma_{K}\right)=\operatorname{Lie}(d \pi(\Gamma))=L(K)$ and

(ii) $\bar{Y}_{x}(\Gamma) \neq\{0\}$ for all $x \in V$.

Proof. If $\Gamma$ is a given subset of $L(G)$, we shall write $G(\widetilde{Y})(x)$ for the orbit of $\bar{Y}(\Gamma)$ through $x \in V$. More precisely, $G(\widetilde{F})(x)$ is the action of the group generated by $\cup_{X \in \mathscr{F}(\Gamma)}\{\exp t X: t \in \mathbf{R}\}$. If $H$ is the subgroup of $G$ generated by

$$
\bigcup_{A \in \Gamma}\{\exp t A: t \in \mathbf{R}\}
$$

at $x$, then $H x=G(\widetilde{y})(x)$. (Here $H x=\{g x+v:(v, g) \in H\}$.)

If $\operatorname{Lie}(\Gamma)=L(G)$, then $H=G$ since $G$ is connected. Thus the orbits of $\widetilde{F}(\Gamma)$ through each point $x \in V$ are given by $G x$. The fact that $K$ acts irreducibly on $V$ implies that $G x \neq\{x\}$ for each $x \in V$. Thus, no orbit of $\widetilde{Y}(\Gamma)$ consists of a single point, and therefore for each $x \in V$ there exists $X \in \bar{Y}(\Gamma)$ such that $X(x) \neq 0$. Thus condition (ii) holds. Since condition (i) is obviously satisfied, we have proved the necessity of our choice.

Conversely suppose that (i) and (ii) hold. Let $\pi_{\Gamma}$ be the restriction of the projection $\pi$ on $\operatorname{Lie}(\Gamma)$. Thus, $\pi_{\Gamma}: \operatorname{Lie}(\Gamma) \rightarrow L(K)$ is a Lie algebra homomorphism. From condition (i) $\pi_{\Gamma}$ is onto. Since $\operatorname{ker} \pi_{\Gamma}$ is an ideal of $\operatorname{Lie}(\Gamma)$, and since $\pi_{\Gamma}$ is onto it follows that $\operatorname{ker} \pi_{\Gamma}$ is a linear subspace of $V$ invariant under $K$. By the irreducibility assumption, either ker $\pi_{\Gamma}=\{0\}$, or ker $\pi_{\Gamma}=V$.

If $\operatorname{ker} \pi_{\Gamma}=V$, there is nothing to prove for then obviously $\operatorname{Lie}(\Gamma)=L(G)$. So assume that $\operatorname{ker} \pi_{\Gamma}=\{0\}$. Thus, $\operatorname{Lie}(\Gamma)$ is isomorphic with $L(K)$. Since $K$ is semisimple and compact it follows that $\operatorname{Lie}(\Gamma)$ is a compact algebra, that is, the integral group $H$ of $\operatorname{Lie}(\Gamma)$ is compact. $H$ being compact, and acting affinely on $V$ has a fixed point. This in turn implies that the orbit of $\mathscr{Y}(\Gamma)$ through this fixed point consists of just that point alone which contradicts our assumption (ii). Thus, $\operatorname{ker} \pi_{\Gamma} \neq\{0\}$ and our proof is now complete.

III. Transitivity of right-invariant systems in the case where $V$ has fixed points under the action of $K$. Before going any further into the details let us consider the following example showing that the Lie algebra condition of the preceding section in the presence of fixed points does not always ensure transitivity.

Example 1. Let $K=S O_{1}(\mathbf{R}) \times S O_{n}(\mathbf{R})$, and let $V=\mathbf{R} \times \mathbf{R}^{n} . K$ acts on $V$ in the obvious way that is $(1, g)(x, y)=(x, g y)$ for each $(1, g) \in K$ and each $(x, y) \in V$. In this action $K v=v$ if and only if $v=(x, 0)$. 
We shall take $G=V \times{ }_{s} K$ and in $G$ we consider a family $\Gamma=\{(v, A): v=(x, y)$, $x>0, A \in L(K)\}$. Then,

(i) $\operatorname{Lie}(\Gamma)=L(G)$ and

(ii) $S(\Gamma)=\{(v, g): v=(x, y): x>0, g \in K\}$.

Hence, $\tilde{\Gamma}_{r}$ is not transitive on $G$ even though $\operatorname{Lie}(\Gamma)=L(G)$.

To elucidate this point we shall assume that $K$ is a given compact group which acts linearly on a finite dimensional vector space $V$. We denote by $\langle$,$\rangle the scalar$ product on $V$ invariant under $K$, and by $d_{V}$ we shall designate the corresponding metric on $V$. Thus, $K \subset O(V)$, where $O(V)$ is the orthogonal group relative to $\langle$,$\rangle .$ If $d_{K}$ denotes the left- and right-invariant metric on $K$, we let $d_{G}$ be the following metric on $G=V \times{ }_{s} K$ :

$$
d_{G}\left(\left(v_{1}, g_{1}\right),\left(v_{2}, g_{2}\right)\right)=d_{K}\left(g_{1}, g_{2}\right)+d_{V}\left(v_{1}, v_{2}\right)
$$

for all $\left(v_{1}, g_{1}\right)$ and $\left(v_{2}, g_{2}\right)$ in $G$. If $(w, h) \in G$, then

$$
\begin{array}{r}
d_{G}\left((w, h)\left(v_{1}, g_{1}\right),(w, h)\left(v_{2}, g_{2}\right)\right)=d_{G}\left(\left(w+h v_{1}, h g_{1}\right),\left(w+h v_{2}, h g_{2}\right)\right) \\
=d_{K}\left(h g_{1}, h g_{2}\right)+d_{V}\left(h v_{1}, h v_{2}\right)=d_{K}\left(g_{1}, g_{2}\right)+d_{V}\left(v_{1}, v_{2}\right) .
\end{array}
$$

Thus, $d_{G}$ is left-invariant.

We let $V_{1}$ be the subspace of $V$ consisting of the fixed points under $K$, and we designate by $V_{2}$ the orthogonal complement of $V$. Since $V_{1}=\{v: K v=v\}$, it follows that for any $X \in L(K), X v=0$ for all $v \in V_{1}$. Moreover, if $w \in V_{2}$, and if $X \in L(K)$, then

$$
\langle v, X w\rangle=-\langle X v, w\rangle=0 \text { for all } v \text { in } V_{1} .
$$

Hence, $X V_{2} \subset V_{2}$. Thus, both $V_{1}$ and $V_{2}$ are invariant under the elements of $L(K)$. We will use $P$ to designate the orthogonal projection of $V$ onto $V_{1}$. As already mentioned earlier, $\tau$ is the canonical projection of $G$ onto $V$, and $d \tau$ is the projection of $L(G)$ onto $V$. We now have the following

THEOREM 3. Let $\Gamma$ be a subset of $L(G)$. Then the associated right-invariant system $\tilde{\Gamma}_{r}$ is transitive on $G$ if and only if $\operatorname{Lie}(\Gamma)=L(G)$, and the positive convex cone $C_{0}(P \circ d \tau(\Gamma))$ spanned by $P \circ d \tau(\Gamma)$ is equal to $V_{1}$.

Proof. We first prove the necessity. If $(a, A) \in \Gamma$, then $(a, A)=\left(a_{1}, 0\right) \oplus\left(a_{2}, A\right)$ where $a_{1}=P a$ and $a_{2}=(I-P) a,\left(a_{1}, 0\right)$ and $\left(a_{2}, A\right)$ commute, hence

$$
\exp t(a, A)=\left(a_{1} t, I\right)\left(a_{2}(t), \exp t A\right) \quad \text { where } a_{2}(t) \in V_{2} \text { for all } t
$$

since $A V_{2} \subset V_{2}$.

It is now clear that if $Y=(b, B)$ is another element of $\Gamma$ then

$$
\begin{aligned}
\exp t_{2}(b, B) \cdot & \exp t_{1}\left(a_{1}, A\right) \\
& =\left(a_{1} t_{1}+b_{1} t_{2}, I\right)\left(b_{2}\left(t_{2}\right)+\left(\exp t_{2} B\right) a_{2}(t), \exp t_{2} B \cdot \exp t_{1} A\right) .
\end{aligned}
$$

Thus, the projection of $S(\Gamma)$ onto $V_{1}$ is equal to the positive convex cone spanned by $P \circ d \tau(\Gamma)$. If $\Gamma$ is transitive, then such a cone must be equal to $V_{1}$.

Conversely assume that $\Gamma$ is such that $\operatorname{Lie}(\Gamma)=L(G)$ and $C_{0}(P \circ d \tau(\Gamma))=V_{1}$. 
Let, as before, $T=\{(v, I):(v, I) \in$ int $S(\Gamma)\}$. From Lemma 2, $T$ is nonempty. If $(v, I) \in T$, then let $w=\int_{K} K \cdot v d \mu$ where $\mu$ is the normalized Haar measure on $K . K w=w$ hence $w \in V_{1}$. If $w=0$, then as in the proof of Theorem 1 it follows that $(0, I) \in T$, and since $G$ is connected, $S(\Gamma)=G$. So in this case there is nothing to prove.

If $w \neq 0$, then there exists a positive integer $N$ such that $v=N w$ is in $T$. This follows from the fact that $w$ belongs to the closed convex cone $C_{0}(K v)$ spanned by the orbit $K v$. Thus, $w=\sum_{i=1}^{p} \lambda_{i} g_{i} v$ for some $g$ elements $g_{1}, \ldots, g_{p}$ in $K$ and positive numbers $\lambda_{1}, \ldots, \lambda_{p}$. By Lemma 4, there exist integers $M_{j}$ such that $\left(M_{j} \lambda_{j} g_{j} v, I\right) \in T$. The desired integer $N$ can then be taken to be equal to $\prod_{j=1}^{p} M_{j}$.

We next show that there exists $\lambda>0$ such that both $\lambda v$ and $-\lambda v$ belong to int $S\left(C_{0}(\Gamma)\right)$ where $C_{0}(\Gamma)$ stand for the convex cone spanned by $\Gamma$. Since $C_{0}(P \circ d \tau(\Gamma))=V_{1}$ there exists an element $X$ of $C_{0}(\Gamma)$ of the form $(-v+u, A)$ where $u \in V_{2}$, and $A \in L(K)$.

$$
\exp t(-v+u, A)=(-v t+u(t), \exp t A) \quad \text { where } u(t) \in V_{2} \text { for all } t .
$$

Since $(v, I) \in T$ which is open it follows that some ball $B(v, \varepsilon)$ of radius $\varepsilon$ centered at $v$ is contained in $T$. From the left-invariance of the metric $d_{G}$ it follows that $B(\exp t X v, \varepsilon)$ is contained in int $S\left(C_{0}(\Gamma)\right)$. Now, $K$ is compact, hence there exists time $t>1$ such that $d_{K}(\exp t A, I)<\varepsilon$. Therefore,

$$
d_{G}(((1-t) v+u(t), I),((1-t) v+u(t), \exp t A))<\varepsilon .
$$

Thus,

$$
((1-t) v+u(t), I) \in B(\exp t X v, \varepsilon)
$$

and hence,

$$
((1-t) v+u(t), I) \in T\left(C_{0}(\Gamma)\right) .
$$

Now, $\int_{K} K((1-t) v+u(t)) d \mu=(1-t) v$, and by a preceding argument it follows that for some positive integer $M, M(1-t) v \in T\left(C_{0}(\Gamma)\right)$. Since $M(1-t)<0$ it follows, by Lemma 3, that there exists $\lambda>0$ sufficiently large that both $\lambda v$ and $-\lambda v$ are in $T\left(C_{0}(\Gamma)\right)$. Since $T\left(C_{0}(\Gamma)\right)$ is a semigroup it follows that $0=\lambda v-\lambda v$ is in $T\left(C_{0}(\Gamma)\right)$ and thus $T\left(C_{0}(\Gamma)\right)$ contains the identity of $G$. This shows that $S\left(C_{0}(\Gamma)\right)=$ $G$, since $G$ is connected. And, now, we are finished, because it is well known that $S\left(C_{0}(\Gamma)\right) \subset \operatorname{cl} S(\Gamma)$ and that $\operatorname{cl} S(\Gamma)=G$ implies that $S(\Gamma)=G$ provided that $\operatorname{Lie}(\Gamma)=L(G)$. Our proof is therefore finished.

IV. Related results and applications. The first result deals with transitivity of systems on semidirect products which are not necessarily defined by compact groups.

THEOREM 4. Let $H$ be a connected Lie group which acts on a finite dimensional vector space $V$, and let $G=V \times{ }_{s} H$. Let $\pi$ be the canonical projection of $G$ onto $H$. Assume that $H$ contains a maximal compact group $K$ which has no nonzero fixed points in $V$. Then, a necessary and sufficient condition that a right-invariant system $\tilde{\Gamma}_{r}$ be transitive on $G$ is that

(i) $\Gamma_{H}=d \pi(\Gamma)$ is transitive on $H$ and

(ii) $\operatorname{Lie}(\Gamma)=L(G)$. 
Proof. The conditions of the theorem are evidently necessary, hence that proof will be omitted. So assume that conditions (i) and (ii) hold. As before, condition (ii) implies that int $S(\Gamma)$ is nonempty. If $(v, g) \in$ int $S(\Gamma)$, then by condition (i) there exists $w \in V$ such that $\left(w, g^{-1}\right) \in S(\Gamma)$. Thus, $(v+G W, I) \in$ int $S(\Gamma)$. Hence, $T$ as defined in the previous lemmas is nonempty. If $(v, I) \in T$ then consider $w=\int_{K} K$. $v d \mu . w$ is invariant under $K$ and hence $w=0$. The rest of the proof follows as in the proof of Theorem 1. Hence, the origin is in $T$, and thus $S(\Gamma)=G$. This proves the theorem.

The following example shows that without any assumption on the compact subgroup $K$ conditions (i) and (ii) do not in general guarantee transitivity.

EXAMPLE 2. Let $H=S O_{0}(n, 1)$ be the connected component through the identity of the Lorentz group in $\mathbf{R}^{n}$. This group, as a subgroup of $G L_{n+1}(\mathbf{R})$, acts linearly on $V=\mathbf{R}^{n+1}$. Consider $G=V \times{ }_{s} H$. Let $C$ be the light cone of $H$ in $V$, and let $\Gamma=C \oplus_{s} L(H)$. Then, obviously, conditions (i) and (ii) are satisfied, but $S(\Gamma)=C$ $\times H \neq G$. In this case, the maximal compact subgroup $K$ of $H$ is equal to $S O_{n}(\mathbf{R}) \times S O_{1}(\mathbf{R})$ which has many fixed points in $V$.

As a way of applications of the preceding results we will concentrate on the following two distinct cases:

Case I. Serret-Frenet moving frames. Recall that a curve $x(s)$ in $\mathbf{R}^{n}$ parametrized by its arc-length from a certain fixed point $x_{0}$ is called regular if $d x / d s$, $d^{2} x / d s^{2}, \ldots, d^{n-1} x / d s^{n-1}$ are linearly independent for each $s>0$. Every regular curve $x$ generates an element $V(s) \in O_{n}(\mathbf{R})$, the so-called moving frame of $x$ at $s$, and it generates $n-1$ curvatures $\kappa_{1}(s), \ldots, \kappa_{n-1}(s)$. If $v_{1}(s), \ldots, v_{n}(s)$ are the columns of $V(s)$, then we have the following well-known Serret-Frenet formulas:

$$
\begin{aligned}
\frac{d x}{d s} & =v_{1}, \\
\frac{d v_{1}}{d s} & =\kappa_{1} v_{2}, \\
\frac{d v_{i}}{d s} & =-\kappa_{i-1} v_{i-1}+\kappa_{i+1} v_{i+1}, \quad 1<i<n, \\
\frac{d v_{n}}{d s} & =-\kappa_{n-1} v_{n-1} .
\end{aligned}
$$

The above set of differential equations can be regarded as a differential system on the semidirect product of $\mathbf{R}^{n}$ with $O_{n}(\mathbf{R})$ : If we regard $g(s)=(x(s), V(s))$ as a curve on $G=\mathbf{R}^{n} \times{ }_{s} O_{n}(\mathbf{R})$ then $d g / d s=g(s) \cdot L\left(\kappa_{1}(s), \ldots, \kappa_{n-1}(s)\right)$ where for each $s>0$, $L\left(\kappa_{1}(s), \ldots, \kappa_{n-1}(s)\right)$ is an element of the Lie algebra of $G$, defined by $L\left(\kappa_{1}(s), \ldots, \kappa_{n-1}(s)\right)=\left(e_{1}, \sum_{i=1}^{n-1} \kappa_{i}(s) A_{i}\right)$ where $A_{i}=-E_{i(i+1)}+E_{(i+1) i}$ for each $i=$ $1,2, \ldots, n-1$. (Here, $E_{i j}=e_{i} \oplus e_{j}$.) We shall assume that $n-2$ curvatures $\kappa_{1}, \ldots, \kappa_{i-1}, \kappa_{i+1}, \ldots, \kappa_{n-1}$ are a priori given and are constant with respect to $s$, and we shall be interested with transitivity results as the remaining $i$ th curvature $\kappa_{i}$ is allowed to vary over all positive smooth functions on $[0, \infty)$. If we let $X=$ $\left(e_{1}, \sum_{j \neq 1}^{n-1} \kappa_{j} A_{j}\right)$ and $Y=\left(0, A_{i}\right)$ then the set of curves $g(s)$ in $G$ generated by the $i$ th curvature $\kappa_{i}$ satisfies

$$
d g / d s=g \cdot\left(X+\kappa_{i} Y\right)
$$


If $\Gamma=\left\{X+\kappa_{i} Y: \kappa_{i}>0\right\}$, then $S(\Gamma)$ consists precisely of all points in $G$ which lie on the solutions of (1) emanating from the identity of $G$ as $\kappa_{i}(s)$ varies over all piecewise constant positive functions on $[0, \infty)$. As explained in $[\mathbf{J}], \operatorname{Lie}(\Gamma)=L(G)$ except in one case. This exceptional case is when $n$ is even, $i=n / 2$ and $\kappa_{i-j}=\kappa_{i+j}$ for all $j=1,2, \ldots, i-1$. When $\operatorname{Lie}(\Gamma)=L(G)$ it then follows from Theorem 1 that $S(\Gamma)=G$ and hence we have the following result:

If $g_{0}$ and $g_{1}$ are any points in $\mathbf{R}^{n} \times S O_{n}(\mathbf{R})$ then there exist an $s_{1}>0$ and a positive (smooth) function $\kappa_{i}(s)$ defined on $\left[0, s_{1}\right]$ such that the corresponding solution $g(s)$ of equation (1) satisfies $g(0)=g_{0}$ and $g\left(s_{1}\right)=g_{1}$ stated differently, for any initial point $x_{0}$ and any initial frame $V_{0}$ in $S O_{n}(\mathbf{R})$ and for any final point $x_{1}$ and final frame $V_{1}$ in $S O_{n}(\mathbf{R})$ there exists a regular curve satisfying:

(i) $x$ satisfies the given curvature constraints and

(ii) it connects the two given points with prescribed initial and final frame.

Case II. Affine control systems on $\mathbf{R}^{n}$. Here we will consider differential control systems on $\mathbf{R}^{n}$ of the form:

$$
\frac{d x}{d t}=A x+a+\sum_{i=1}^{m} u_{i}(t)\left(B_{i} x+b_{i}\right)
$$

where $A, B_{1}, \ldots, B_{m}$ are $n \times n$ matrices, and where $a, b_{1}, \ldots, b_{m}$ are vectors in $\mathbf{R}^{n}$. The state variable $x$ is in $\mathbf{R}^{n}$ while the controls $u_{1}, \ldots, u_{m}$ each takes values in $\mathbf{R}$ and is defined for $t \in[0, \infty)$. We call such systems affine. For simplicity of exposition, we will concentrate only on the case of scalar control:

$$
\frac{d x}{d t}=A x+a+u(t)(B x+b) .
$$

The system defined by (3) is said to be (completely) controllable if for any initial state $x_{0}$ and any final state $x_{1}$ in $\mathbf{R}^{n}$ there exists a control $u$ such that the corresponding solution of (3) starts at $x_{0}$ at $t=0$ and passes through $x_{1}$ in some positive time $t$. When $a=0$ and $B=0$, then such a system is called linear and as is well known in the literature of control theory that controllability is equivalent to $b, A b, \ldots, A^{n-1} b$ being linearly independent.

We will however regard equation (3) as a part of a larger system defined as follows:

Assume that the admissible controls $u$ are required to take values in some constraint set $\Omega \subset \mathbf{R}$. We shall regard $A$ and $B$ to be elements of $M_{n}(\mathbf{R})$ the Lie algebra of $G L_{n}(\mathbf{R})$ the general linear group of $\mathbf{R}^{n}$. As such the set $\{A+u B: u \in \Omega\}$ generates a certain Lie subalgebra of $M_{n}(\mathbf{R})$. We denote by $L$ such a subalgebra, and we denote the integral group of $L$ by $H$. $H$ is the group generated by

$$
\bigcup_{M \in I .}\left\{e^{M t}: t \in \mathbf{R}\right\} \text {. }
$$

We regard $X=(a, A)$ and $Y=(b, B)$ to be elements of the Lie algebra of $G=\mathbf{R}^{n} \times_{s} H$. Instead of just equation (3) we consider

$$
d g / d t=(X+u(t) Y) g(t), \quad g(t) \in G .
$$

If $g(t)=(x(t), h(t))$ then $x$ satisfies equation (3) while $h$ satisfies

$$
d h / d t=(A+u(t) B) h(t) .
$$


When the admissible controls vary over all piecewise constant functions defined on $[0, \infty)$ with values in $\Omega$, then as is well known, the set of points in $G$ reachable from the identity via the solutions of equation (4) is given by $S(\Gamma)$ where $\Gamma=\{X+$ $u Y: u \in \Omega\}$.

Transitivity of $\tilde{\Gamma}$ on $G$ implies in particular controllability of equation (3). From our choice of $H$ we already know that $\Gamma_{H}=d \pi, \operatorname{Lie}\left(\Gamma_{H}\right)=d \pi(\operatorname{Lie}(\Gamma))$ is equal to $L(H)$ so in order to apply the preceding transitivity results we need to verify that

(i) either $H$ is compact, or that $\Gamma_{H}$ is transitive on $H$, and

(ii) that $\operatorname{Lie}(\Gamma)=L(G)$.

The results contained in $[\mathbf{J K}]$ deal with conditions $A$ and $B$ which ensure that equation (5) is controllable or, equivalently, that the corresponding right-invariant system $\tilde{\Gamma}_{H}$ is transitive on $H$.

A more detailed analysis of affine systems using the semidirect products of Lie groups will appear elsewhere.

\section{REFERENCES}

[J] V. Jurdjevic, On the reachability properties of curves in $\mathbf{R}^{n}$ with prescribed curvatures, University of Bordeaux Publ. 1, no. 8009, 1980.

[JK] V. Jurdjevic and I. Kupka, Control systems subordinated to a group action: Accessibility, J. Differential Equations 38 (1980).

[JS] V. Jurdjevic and H. Sussmann, Control systems on Lie groups, J. Differential Equations 12 (1972), 313-329.

[K] A. J. Krener, A generalization of Chow's theorem and the Bang-Bang theorem to non-linear control problems, SIAM J. Control Optim. 1 (1974), 43-51.

[L] C. Lobry, Controllability of non-linear. systems on compact manifolds, SIAM J. Control Optim. 1 (1974), 1-4.

[SJ] H. Sussmann and V. Jurdjevic, Controllability of non-linear systems, J. Differential Equations 12 (1972), 95-116.

Department of Mathematics, University of Bordeaux, Bordeaux, France

Department of Mathematics, University of Toronto, Toronto, Ontario M5S 1A7, Canada

Department of Mathematics, University of Grenoble, Grenoble, France

Department of Mathematics, University of Metz, Strasbourg, France 